
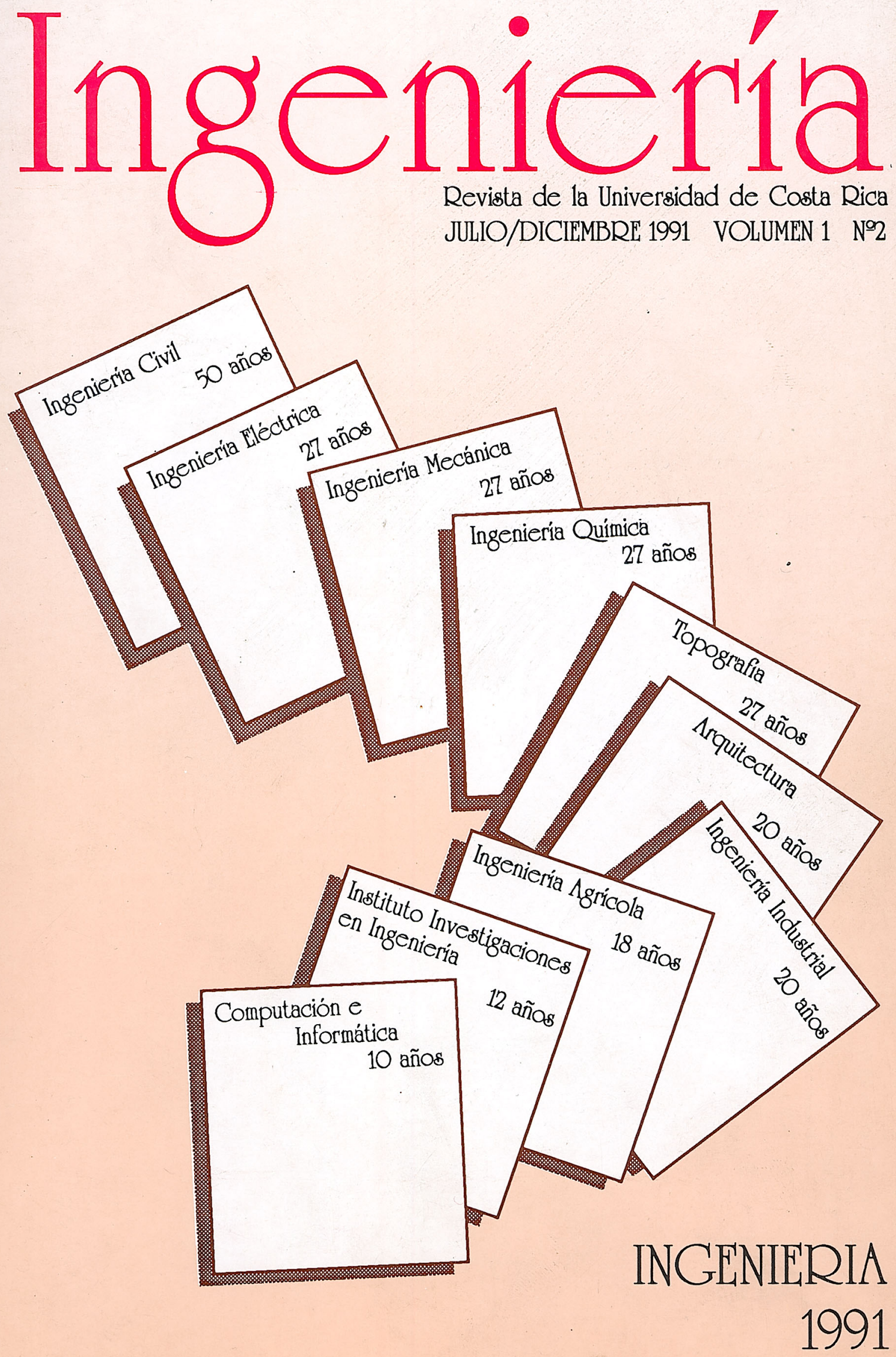


\title{
TRABAJOS FINALES DE GRADUACION DE LA FACULTAD DE INGENIERIA. GRADUACIONES DE 1990
}

\begin{abstract}
Se presentan en esta sección las Referencias Bibliográficas de los Trabajos Finales de Graduación de la Facultad de Ingenieria de la Universidad de Costa Rica las graduaciones del año 1990. Comprende además del listado general de los trabajos, los resúmenes de los de mayor relevancia en este periodo.
\end{abstract}

\section{ESCUELA DE INGENIERIA AGRICOLA}

1 Semestre de 1990

GUILLEN FUENfES, ARTURO.

Evaluación del Sistema de Secado y Puesta en Marcha del Sistema de Secaireación en Planta Térraba.

Tesis de Ingeniería Agrícola. San José, C.R: A Guillén F., 1990

MONTERO PEREIRA, LUIS M. Y RODRIGUEZ SANDOV AL, GISELLE.

Evaluación de la Conductividad Hidráulica de Cinco Parcelas Experimentales y su Aplicación a una Red de Drenaje en Banano.

Tesis de Ingeniería Agrícola. San José, C.R:

L. M. Montero P. y G. Rodríguez S., 1990

ZAMORA MIRANDA, RICARDO.

Análisis del Secado de Capa Fija en Café Pergamino (Coffea arabica) en Silo Metálico con Piso Falso.

Tesis de Ingeniería Agrícola. San José, C.R:

R Zamora M., 1990.

\section{Semestre de 1990}

CARMONA ESTRADA, ISMAEL.

Sistema de Riego para Santa Barbara de Heredia.

Tesis de Ingeniería Agrícola. San José, C.R:

1. Carmona E., 1990.

HENDERSON BLACKMAN, BELINDA

Un Análisis de Prefactibilidad para Establecer una Planta de Procesamiento de Frijol en Costa Rica.

Tesis de Ingeniería Agrícola. San José, C.R:

B. Henderson B.,1991.

MATA ACUÑA, GUSTAVO.

Modelos de Calibración para Equipos de Medición de Contenido de Humedad en Café (Coffea arabica).

Tesis de Ingeniería Agrícola. San José, C.R:
G. Mata A, 1990 .

SANCHEZ CORDOBA, FERNANDO.

Un Modelo de Balance Hídrico Computarizado'

Tesis de Ingeniería Agrícola. San José, C.R:

F. Sánchez C., 1991.

\section{RESUMENES}

SANCHEZ CORDOBA, FERNANDO.

Un Modelo de Balance Hídrico Computarizado.

Tesis de Ingeniería Agrícola. San José, C.R:

F. Sánchez C., 1991.

El proyecto de graduación consiste en la programación para una computadora compatible con la IBM-XT de un modelo de balance hídrico, y fue realizado con el apoyo y lineamientos del proyecto de investigación "Optimización Hídrica de la Agricultura Tropical de Secano", financiado por la Agencia para el Desarrollo Internacional (ALD.) de los E.E.U.U. y efectuado en la Escuela de Ingeniería Agrícola de la Universidad de Costa.

El modelo de balance y el programa obtenido en este proyecto se ajustan mejor que otros programas a las características climáticas de la zona tropical y a los pocos datos que se pueden obtener en estas zonas. Esto se debe primordialmente a que el programa hace uso de valores diarios de precipitación y valores mensuales de evapotranspiración potencial, lo que se ajusta a la gran variabilidad de la precipitación diaria y a la relativamente poca variabilidad de la evapotranspiración potencial.

A partir del cálculo del balance hídrico, el programa es capaz de estimar la fecha más temprana de siembra para un cultivo, la última fecha en que el suelo está a capacidad de campo en el año y el número de días con déficit, exceso o estrés hídricos. A partir de los cálculos el programa puede generar reportes impresos, por pantalla o a disco (archivos ASCII). 
El balance hídrico y los cálculos relacionados son de gran utilidad para el manejo de una zona, pues permiten identificar las potencialidades que ella posee desde el punto de vista hídrico.

Director de Tesis: Dr. Ricardo Raldulovich R

\section{ESCUELA DE INGENIERIA CML}

\section{Semestre de 1990}

\section{ANDERSON HERRERA AUDREY.}

Optimización de las dimensiones de urbanizaciones de Carácter Social.

Tesis de Ingeniería Civil. San José, C.R:

A Anderson H., 1990.

ANGLIN FONSECA ROBERT.

Análisis de alternativas ydiseño preliminar de la Intersección "La Datsun".

Tesis de Ingeniería Civil. San José, C.R:

R Anglin F., 1990.

ARCE GUZMAN JOSE ROBERTO.

Problemática de las inundaciones en Costa Rica. Una propuesta de análisis.

Tesis de Ingeniería Civil. San José, C.R:

J. R Arce G., 1990.

ARGUEDAS MORA CARLOS FERNANDO.

Optimización de redes hidráulicas.

Tesis de Ingeniería Civil. San José, C.R:

C. F. Arguedas M., 1990.

\section{BLANCO RODRIGUEZ FRANCISCO JAVIER}

Recomendaciones geométricas para autobuses urbanos.

Tesis de Ingeniería Civil. San José, C.R:

F. J. Blanco R, 1990.

\section{CASTILLO BARAHONA ROLANDO.}

Diseño sismoresistente de un marco de concreto reforzado utilizando desplazamientos máximos inelásticos.

R. Castillo B.. 1990.

CHUY SAM CECILIA,

\section{NEUROHR BUSTAMANTE ERICH.}

Estudio del comportamiento de edificios irregulares en elevación.

Tesis de Ingeniería Civil. San José, C.R:

C. Chuy S., 1990.

GRANT SAENZ MARIO.

Uso de la coordinación modular para el diseño yconstrucción de viviendas económicas en Costa Rica.

Tesis de Ingeniería Civil. San José, C.R:

M. Grant S., 1990.

LACHNER NIKLITSCHEK MARIA TERESA

Alternativas de reciclaje en plásticos, papel y vidrio.

Tesis de Ingeniería Civil. San José, C.R:

M.T. Lachner N., 1990.

MARIN JENKINS FAUSTO.

Consideraciones en el diseño sismoresistente de tanques circulares de acero.

Tesis de Ingeniería Civil. San José, C.R:

F. Marín J., 1990.

MENDOZA MORA HECTOR,

VILLALOBOS ARGÜELLO CARLOS.

Capacidad estructural de paneles de bambú.
Tesis de Ingeniería Civil. San José, C.R:

H. Mendoza M., 1990.

MESSEGUER ARMIJO MELANIA.

Diseño sísmico de un marco con muro de corte por desplazamientos inelásticos.

Tesis de Ingeniería Civil. San José, C.R:

M. Messeguer A, 1990.

NAVAS CARRO ALEJANDRO.

Estudio experimental a largo plazo en vigas de concreto reforzadocon bambú.

Tesis de Ingeniería Civil. San José, C.R:

A Navas C., 1990.

RAMIREZ LANDA VERDE DOUGLAS.

Análisis de métodos de reajuste del Banco Hipotecario de la Vivienda.

Tesis de Ingeniería Civil. San José, C.R:

D. Ramírez L., 1990.

SOLIS SOLIS ROSAIRA.

Aplicación del modelo de simulación de obras públicas (SIMOP) a las mejoras del acueducto de Alajuela.

Tesis de Ingeniería Civil. San José, C.R:

R Salís S., 1990.

WIEN SCHECHNER ABRAHAM.

Análisis del divorcio entre las actividades de diseño y construcción en conjuntos habitacionales.

Tesis de Ingeniería Civil. San José, C.R:

A Wien S., 1990.

ZUÑIGA LUNA IRENE.

Problemática de las inundaciones en Costa Rica. Una propuesta de análisis.

Tesis de Ingeniería Civil. San José, C.R:

1.Zúñiga L., 1990.

\section{Semestre de 1990}

ABELLAN ARROYO ALVARO

Estudio comparativo para el cálculo de curvas de deceleraciónen Costa Rica

Tesis de Ingeniería Civil. San José, C.R:

A Abellán A, 1990

ALFARO ARRIETA MARCO AURELIO

Alcantarillado sanitario y pluvial para Río Claro de Golfito Estudio de prefactibilidad.

Tesis de Ingeniería Civil. San José, C.R:

M. A Alfara A, 1990.

CAMPOS CHAV ARRIA CARLOS FRANCISCO

Estudio de pilotes sometidos a carga lateral

Tesis de Ingeniería Civil. San José, C.R:

C. F. Campos Ch., 1990.

CAMPOS VARGAS ERICK, LANZONI RODRI-

GUEZALVARO,

LOPEZ RODRIGUEZ JOSE, PORRAS CORDERO

WILLIAM,

VINDAS PEÑARANDA ALVARO ENRIQUE, VIETO ZELEDON JUAN BAUTISTA

Seminario sobre Régimen Municipal. Mantenimiento de caminos,catastro municipal, servicios municipales.

Tesis de Ingeniería Civil. San José, C.R:

E. Campos V. e.a., 1990.

CASTRO CHA VARRIA RODOLFO

Consumo máximo instantáneo en redes de distribución de agua potable en edificios de oficinas y apartamentos 
Tesis de Ingeniería Civil. San José, C.R:

R Castro Ch., 1990.

\section{CHA VARRIA TORREALBA SILVESTRE}

Programa para diseño de placas combinadas de dos columnas

Tesis de Ingeniería Civil. San José, C.R:

S. Chavarría., 1990.

CHINCHILLA MURILLO RICARDO

Metodología para desarrollar planes maestros de alcantarillado sanitario

Tesis de Ingeniería Civil. San José, C.R:

R Chinchilla M., 1990.

\section{ESPINOZA BLANCO EDWIN}

Mezclas de micro concreto para modelos estructurales Tesis de Ingeniería Civil. San José, C.R:

E. Espinoza B., 1990.

GOMEZ NAVARRO ANA ISABEL

Protección de vidas humanas en caso de incendio

Tesis de Ingeniería Civil. San José, C.R:

A 1. Gómez N., 1990.

\section{GONZALEZ ALPIZAR LUIS}

Evaluación del cumplimiento de las normas de armado paraviviendas en mampostería en 15 construcciones del Area Metropolitana.

Tesis de Ingeniería Civil. San José, C.R:

L. González A, 1990.

GUEV ARA VEGA FIDEL

Proyecto Nacional de Bambú: Evaluación de campo y recomendaciones para mejorar el proceso de construcción

Tesis de Ingeniería Civil. San José, C.R:

F. Guevara V., 1990.

HERNANDEZ HERRERA ROLANDO JOSE

Instrumentación extensiva para medición de respuesta sísmica en un edificio alto.

Tesis de Ingeniería Civil. San José, C.R:

R J. Hernández H., 1990.

\section{LAURENT CUBILLO MAURICIO}

Efecto de escaleras en el comportamiento sísmico de edificios.

Tesis de Ingeniería Civil. San José, C.R:

M. Laurent C., 1990.

MEZA GUTIERREZ LUIS F.

Estudio preliminar para la disposición de aguas residuales de la Ciudad de Golfito

Tesis de Ingeniería Civil. San José, C.R:

L. F. Meza G., 1990.

MILANES MENDEZ JORGE E.

Nuevas fuentes de agregados para la producción de concreto de mediana resistencia

Tesis de Ingeniería Civil. San José, C.R:

J. E. Milanés M., 1990.

\section{MORA JIMENEZ EDUARDO}

Estudio de accidentes de tránsito en el campus universitario Rodrigo Pacio

Tesis de Ingeniería Civil. San José, C.R:

E. Mora 1..., 1990.

\section{MORALES TORRES JAVIER}

Cálculo de muros de contención con ordenadores

Tesis de Ingeniería Civil. San José, C.R:

J. Morales T., 1990.
MOYA LACAYO ALEXANDER

SOLANO RAMIREZ MARIA CRISTINA

Degradación potencial de materiales para la construcción de pavimentos

Tesis de Ingeniería Civil. San José, C.R:

A Moya L. YM. C. Solano R, 1990.

RODRIGUEZ PACHECO MARTIZA

Efectos del desvío del Río Ocloro hacia el Río María Aguilar

Tesis de Ingeniería Civil. San José, C.R:

M. Rodríguez P., 1990.

RODRIGUEZ SOLANO HERIBERTO

Manual para presupuesto de edificaciones

Tesis de Ingeniería Civil. San José, C.R:

H. Rodríguez S., 1990.

SAAVEDRA REYES JOSE ANTONIO

Estudio para un tren suburbano en Puntarenas

Tesis de Ingeniería Civil. San José, C.R:

J. A Saavedra R, 1990.

SALAS CALDERON JORGE EDUARDO

VARGAS BENA VIDES OSCAR

Diseño de una estructura espacial de bambú

Tesis de Ingeniería Civil. San José, C.R:

J. E. Salas C. y O. Vargas B., 1990.

SALAS PEREIRA RUBEN MARIO

Planificación y control de obra Apartotel Punta Leona

Tesis de Ingeniería Civil. San José, C.R:

R M. Salas P.., 1990.

\section{SANCHO MADRIZ LUIS MAURICIO}

Evaluación del cumplimiento de normas de detalle de refuerzo en edificios de concreto reforzado

Tesis de Ingeniería Civil. San José, C.R:

L. M. Sancho M.., 1990.

\section{SAUMASOLANOLAURA}

Evaluación preliminar del acueducto de San Isidro de Heredia

Tesis de Ingeniería Civil. San José, C.R:

L. Sauma S., 1990.

TAPIA BALLADARES MARCO A

Estabilización de arcillas expansivas

Tesis de Ingeniería Civil. San José, C.R:

M. A Tapia B., 1990.

VALVERDE ZUNIIGA FERNANDO

Selección y diseño preliminar de estructura "Paso catarata"Camino de acceso a casa de máquinas. Proyecto Toro II, ICE.

Tesis de Ingeniería Civil. San José, C.R:

F. Valverde Z., 1990.

\section{RESUMENES}

CAMPOS VARGAS ERICK, LANZONI RODRIGUEZALVARO,

LOPEZ RODRIGUEZ JOSE, PORRAS CORDERO WILLIAM,

VINDAS PENAARANDA ALVARO ENRIQUE, VIETO ZELEDON JUAN BAUTISTA

Seminario sobre Régimen Municipal. Mantenimiento de caminos,catastro municipal, servicios municipales.

Tesis de Ingeniería Civil. San José, C.R:

E. Campos V. e.a., 1990. 
Se presenta una visión global del proceso administrativo y sus componentes: la planeación, la organización, la dirección y el control, relacionándolos con las funciones municipales y estableciendo las deficiencias sobresalientes en el sistema.

El análisis se identifica con el punto de vista público y destaca la importancia que tienen los gobiernos locales en el desarrollo de su cantón

Costa Rica cuenta con ochenta y un cantones cada uno regido por un gobierno local. Existen por lo tanto ochenta y un Municipalidades Cantonales y un Distrito Municipal (Hatillo).

El presente estudio clasifica las municipalidades en rurales, semiurbanas y urbanas con base en los siguientes parámetros: ingreso municipal, superficie del cantón, densidad de población, ingreso por área y por población. A continuación viene un escogimiento de varias municipalidades de cada clase para tener la lista de posibles municipalidades modelo y municipalidades proyecto: $\mathrm{Ru}-$ ral: Orotina, El Guarco, San Isidro de Heredia; Semiurbano: Aserrí, Grecia, Santa Ana, Barva, Vásquez de Coronado; Urbano: Goicoechea, Heredia, Tibás, Santo Domingo.

Inicialmente se pensaba tener una municipalidad proyecto de cada clase, pero el escaso tiempo y los problemas logísticos redujeron esto a una sola, que fue escogida del grupo rural y correspondió a San Isidro de Heredia.

Municipalidad proyecto es la que tiene deficiencias en los campos seleccionados para el presente estudio, que fueron mantenimiento de caminos, catastro municipal, sistema de dirección postal y sistema pluvial.

Municipalidades modelo son las que tienen resueltos satisfactoriamente algunos de los aspectos citados y, como tales, constituyen magníficas referencias y guías para los problemas a resolverle a la municipalidad proyecto. Fueron utilizadas Grecia, Pérez Zeledón y Santa Ana como municipalidades modelo (en uno o varios campos).

Se señalan las características generales de San Isidro de Heredia, cantón escogido como proyeto.

Se presenta el inventario de los caminos del cantón, siguiendo la modalidad del MOPT Ministerio de Obras Públicas y Transportes y utilizando su información. Se dan normas y recomendaciones para su mantenimiento. Sobre este aspecto de mantenimiento de caminos se incluye un manual con las labores a realizar por las municipalidades e ideas para obtener los recursos necesarios para desarrollarlas.

Se realiza un diagnóstico del estado actual del catastro de San Isidro de Heredia, proponiendo algunas reformas ingenieriles. También se suministra un manual que contiene tantó el procedimiento como fichas para el levantamiento y el proceso de la información de campo. Se propone un sistema para dirección postal, que utiliza como referencia calles, avenidas y caminos.

Se analizan los servicios prestados por la mayor parte de las municipalidades, con énfasis en los ofrecidos por la municipalidad proyecto. Se incluye el diagnóstico actual del alcantarillado pluvial, una propuesta para su mejoramiento y algunas ideas sobre conservación de cuencas hidrográficas, especialmente las que suplen el agua potable del cantón.

Comité Asesor: Oscar Delgado, Miguel Dobles, Enrique Rodríguez, Francisco Vargas.

\section{ESCUELA DE INGENIERIA ELECTRICA}

\section{Semestre de 1990}

SIBAJA, ALDO.

Filtro Digital de Llamadas.

Tesis de Ingeniería Eléctrica (Bach.). San José, C.R:

A. Sibaja, 1990

\section{GUILLEN GARCIA, LUDWING}

Diseño de una Máquina de Clasificación de Arrancadores para Fluorescentes

Tesis de Ingeniería Eléctrica (Baéh.). San José, C.R:

L. Guillén G., 1990.

ARAUZ CENTENO, MARCO LIBIO Y SILESKY SOTO, CARLOS MANUEL.

Normas de las Instalaciones Eléctricas en Viviendas de Interes Social.

Tesis de Ingeniería Eléctrica (Bach.). San José, C.R M. L. Arauz C. Y C. M. Silesky S., 1990.

\section{ESQUIVELSEDO, FABIAN.}

Diseño de una Mesa de Pruebas para el control de Calidad en Lámparas Fluorescentes.

Tesis de Ingeniería Eléctrica (Bach.). San José, C.R:

F. Esquivcl S., 1990.

\section{VICTOR SANCHEZ,LUIS FERNANDO.}

Diseño de control Digital Programable de Procesos Movidos por Motores eléctricos.

Tesis de Ingeniería Eléctrica (Bach.). San José, c.R.:

L. F. Victor S., 1990

CHA VES VARGAS, RICARDO Y MURILLO RAMIREZ, DANILO.

Automatización de un Sistema Auxiliar de Aire Acondicionado Utilizando un controlador Lógico Progamable. Tesis de Ingeniería Eléctrica (Bach.). San José, C.R: R Chaves V.y .D. Murillo R, 1990.

LONGAN PHILLIPS, GINA.

Programa para la Simulación de un Protocolo de Enlaces de Datos.

Tesis de Ingeniería Eléctrica (Bach.). San José, c.R. G. Longan P., 1990.

\section{MONTURIOLLE ROY, JOSE y VENEGAS MONGE, GILBERT.}

Laboratorio de Mantenimiento para Microcomputadoras del I.C.E.

Tesis de Ingeniería Eléctrica (Bach.). San José, c.R.: J. Monturiol L. R YG. Venegas M., 1990. 
CALVO HORTH, LUIS GUSTAVO.

Progama de Diseño del Devanado de Arranque de un Motor Monofásico para uso en una Computadora Personal.

Tesis de Ingeniería Eléctrica (Bach.). San José, C.R:

L. G. Calvo H., 1990.

JIMENEZ SANCHO, PAOLA.

Elaboración de las Herramientas Básicas para la Creación del Compilador del Lenguaje FACIL.

Tesis de Ingeniería Eléctrica (Bach.). San José, C.R:

P. Jiménez S.. 1990.

VARGAS RODRIGUEZ, JOSE ALBERTO y GARCIA VARGAS, MAURICIO.

Sistema de Desarrollo Basado en el Microcontrolador $8051 / 8052$.

Tesis de Ingeniería Eléctrica (Bach.). San José, C.R:

J. A. Vargas R y M. García V., 1990.

\section{CHENG LO, YIN HO y KUHLMANN BERENZON,} HANS.

Sistema de Desarrollo Basado en el Microprocesador 8088 para IBM-XT ó Compatible.

Tesis de Ingeniería Eléctrica (Bach.). San José, C.R:

Y. H. Cheng. y H. Kuhlmann B., 1990.

\section{PORRAS VARGAS, ROGERE.}

Unidad de Adquisición de Datos para el Microprocesador MC 68000.

Tesis de Ingeniería Eléctrica (Bach.). San José, C.R:

R Porras V., 1990.

\section{MOYA VARGAS, LUIS.}

Diseño de un controlador Automático y Programable de Máxima Demanda de Energía Eléctrica

Tesis de :ngeniería Eléctrica (Bach.). San José, C.R:

L. Moya V., 1990.

\section{MEJIAS UGALDE, RONALD.}

Estudio de Necesidades de Equipo y Soporte Técnico para la Comunicación de Datos en el Sector Público Costarricense

Tesis de Ingeniería Eléctrica (Bach.). San José, C.R:

R Mejías U., 1990.

\section{SANCHO CHA VES, JORGE LUIS}

Ampliación de la Planta Hidroeléctrica de Carrillo de Poas: Estudio de Factibilidad.

Tesis de Ingeniería Eléctrica (Bach.). San José, C.R:

J. L. Sancho CH.

\section{CASTRO SOLERA, MAX.}

Control del factor de Potencia en Grandes Cargas Fluctuantes.

Tesis de Ingeniería Eléctrica (Bach.). San José, C.R:

M. Castro S., 1990.
MORA MONGE, HANSEL MANUEL y VARGAS GARCIA, FELIPE A.

Ajuste finales y Ensamble de una fuenste de Alimentación y Diseño y Construcción de Generador de Timbrado para un Central Telefónica Digital H. M. Mora M. y F. A. Vargas G., 1990.

\section{CAMPOS LOPEZ, CARLOS.}

Desarrollo de un Sistema de Control; por Medio de una Computadora IBM-XT para el Robot Hidráulico de la Escuela de Ingeniería Eléctrica de la U.C.R

Tesis de Ingeniería Eléctrica (Lic.). San José, C.R: C. Campos L.

\section{Semestre de 1990}

MESEGUER ARMIJO, EDGAR ANTONIO y SANCHO RUBI, VICTOR JULIO.

Programador de Memoria EPROM y EEPROM "Proms1".

Tesis de Ingeniería Eléctrica (Bach.). San José, C.R: E. A. Meseguer A. y V. J. Sancho R, 1990.

MONGE CORDERO, LARISA.

Sistema Fotodetector Mejorado Uti! para Medir y Graficar Potencia Optica.

L. Monge C., 1990.

\section{VARELA RODRIGUEZ, JAVIER}

Metodos de Trabajo en Subestaciones y Características de la Red de Distribución de la Compañía Nacional de Fuerza y Luz S.A

Tesis de Ingeniería Eléctrica (Bach.). San José, C.R: J. Varela R, 1990.

VEGA PORRAS, LAURENCE, y MANGEL HEREDIA, CRISTIAN.

Sondeo Sobre las Necesidades de Equipo para la Transmisión de Datos en Empresas Privadas del Valle Central. Tesis de Ingeniería Eléctrica (Bach.). San José, C.R: L. Vega P. y C. Mangel H., 1990.

\section{PEREZ BARRIENTOS, JOHNNY.}

Diseño y Construcción de un Prototipo de Analizador de Datos para Transmisión de Información Vía Telefonica. Tesis de Ingeniería Eléctrica (Bach.). San José, c.R.: J. Pérez B., 1990.

FERNANDEZ FLORES, W.

Perforador Inteligente de Tarjetas de Circuitos Impresos. Tesis de Ingeniería Eléctrica (Bach.). San José, C.R: W. Fernandez F., 1990.

\section{CHA VES, JULIO CESAR}

Motores de Pasos: Teoria General y Diseño de un Controlador.

Tesis de Ingeniería Eléctrica (Bach.). San José, C.R: J. C. Chaves, 1990.

YANG TSE-HUA, LAN

Construcción y Optimización de un Modulo Estabilizado de Pruebas para Diodos LASER 
Tesis de Ingeniería Eléctrica (Bach.). San José, C.R: L. YangT.H., 1990.

SANCHO ARROYO, HUGO.

Bastidor de una Central Telefónica Digital Privada PABX

Tesis de Ingeniería Eléctrica (Bach.). San José, C.R: H. Sancho A, 1990.

\section{UREÑA GRANADOS, GUILLERMO.}

Diseño y Construcción de la Tarjeta Impresa de los Circuitos de Unea para una Central Telefónica Digital PrivadaPABX

Tesis de Ingeniería Eléctrica (Bach.). San José, C.R: G. UreñaG .

\section{ACUÑA, ENRIQUE Y LUNA, ALEJANDRO.}

Diseño Lógico de la CPUCR

Tesis de Ingeniería Eléctrica (Bach.). San José, C.R:

E. Acuña y A Luna, 1990.

\section{CHACON CASTRO, JORGE EDUARDO}

Diseño y Construcción de un Control Electronico de Frecuencia para Microcentrales Hidroeléctricas.

Tesis de Ingeniería Eléctrica (Bach.). San José, C.R: J. E. Chacón C., 1990.

\section{ORTIZ RAMIREZ, WILLIAM ALBERTO.}

Diseño y Construcción de un Controlador de Velocidad para un Motor C.D. Utilizando un Sistema PLL. Tesis de Ingeniería Eléctrica (Bach.). San José, C.R: W.A OrtizR

ANDRE TINOCO, ALEX y MENDEZ VIQUEZ, ROGER

Identificación de la Ecuación en Diferencias de una Planta Simuladora con el Computador Analógico de la Escuela de Ingeniería Eléctrica.

Tesis de Ingeniería Eléctrica (Lic.). San José, C.R:

A André T. y R Méndez V.

ARROYO A VILA, EDGAR; RAMIREZ CALDERON, WILLIAM Y RODRIGUEZ ZAMORA, IV AN. Análisis de Ahorro energético del Sector Residencial en el Campo de la Iluminación.

Tesis de Ingeniería Eléctrica (Lic.). San José, C.R: E. Arrollo A, e.a., 1990.

\section{DELGADO CASCANTE, GEOV ANNY A}

Diseño y Construcción de un Sistema Digital de Control de Riego.

Tesis de Ingeniería Eléctrica (Lic.). San José, C.R:

G. A Delgado C., 1990

FUNG HO, JOSE J.

Análisis y Simulación del diagrama Digital de Estados. Tesis de Ingeniería Eléctrica (Lic.). San José, C.R: J. J. Fung H., 1990.
PANIAGUA CARRANZA, CLAUDIO D. y MORA ROJAS, FREDDY J.

Estudio Sobre el Estado de Instalaciones Eléctricas Residenciales en la Provincia de San José.

Tesis de Ingeniería Eléctrica (Lic.). San José, C.R:

C. D. Paniagua C. y F. J. Mora R, 1990.

\section{RESUMENES}

CAMPOS LO PEZ, CARLOS.

Desarrollo de un Sistema de Control, por Medio de una Computadora IBM-XT para el Robot Hidráulico de la Escuela de Ingeniería Eléctrica de la U.C.R

Tesis de Ingeniería Eléctrica (Lic.). San José, C.R:

C. CamposL.

Se diseño y construyó una tarjeta para el computador a manera de interface y se desarrollo un programa para manejar la tarjeta y facilitar al usuario común el movimiento del robot sin contar con amplios conocimientos de robótica ó programación.

Director de Tesis: Ing. Jorge E. Badilla.

DELGADO CASCANTE, GEOV ANNY A

Diseño y Construcción de un Sistema Digital de Control de Riego.

Tesis de Ingeniería Eléctrica (Lic.). San José, C.R:

G. A Delgado C., 1990.

El sistema digital de control de riego es un equipo desarrollado con un microcontrolador. Este equipo tiene la capacidad de controlar 16 válvulas para un programa de riego temporizado, donde se definen los tiempos de válvula abierta y de válvula cerrada. El sistema es programable, de tal forma que, mediante una subrutina que interactúl con el usuario através de una pantalla y un teclado, es posible ingresar en el sistema: tiempos de válvula abierta y cerrada, modo de operación, inicia en válvula abierta, inicio en válvula cerrada, etc.

El sistema es multitarea en tiempo real, tal que el equipo puede ejecutar más de una tarea en forma simultánea; el usuario puede revisar el programa para una válvula o reprogramarla sin detener la ejecución normal del programa. El equipo se diseño y construyó y pronto estará listo para una fase de pruebas en el campo.

Director de Tesis: Ing. Guillermo Loría Mart\{nez.

\section{ESCUELA DE INGENIERIA INDUSTRIAL.}

\section{$1 Y$ 11Semestres de 1990.}

SOLANO VILLA VERDE, CARLOS.

Evaluación económica de un proyecto forestal. Tesis de Ingeniería Industrial. San José, C.R: C. Solano V., 1990.

DURAN CALVO, LUZ ESTHER Y MANTILLA LOPEZ, JEAN DESIREE.

Sistema de desarrollo de proveedores, RECOPE.

Tesis de Ingeniería Industrial. San José, C.R: 
LE. Durán C. y J. D. Mantilla L., 1990.

PEREZ GARCIA, LEIDY y ARANA ABREL, CARLOS.

Diseño de un:sistema de mantenimiento y recuperación de equipo y maquinaria utilizados en los cursos que imparte el INA

Tesis de Ingeniería Industrial. San José, C.R:

L. Pérez G. y C. Arana A, 1990.

OROZCO SABORIO, KEVIN y BENA VIDES HERNANDEZ, LUIS ARMANDO.

Sistema para la normalización de los materiales utilizados en los cursos del INA

Tesis de Ingeniería Industrial. San José, C.R:

K Orozco S. y L. ABenavides H., 1990.

MORERA PACHECO, JUAN MIGUEL Y MORERA SOLORZANO, LUIS ALBERTO.

Diagnóstico del sistema de manejo de la ropa del Hospital Dr. Rafael A Calderón Guardia y Propuestas para su mejoramiento

Tesis de Ingeniería Industrial. San José, C.R:

J. M. Morera P. y L. A Morera S., 1990.

BERMUDEZ GOMEZ, HUGOj CHASSOUL ACOSTA, MARIA JOSE y YONG AGUILAR, MANUEL ENRIQUE.

Dianóstico metrológico de la Universidad de Costa Rica

Tesis de Ingeniería Industrial. San José, C.R;

H. Bermúdez G. et al., 1990.

CHONGKAN CH., FREDDY y ARCE ACHI, MINOR Compras del Estado: metodología para la determinación de temas con prioridad de normalización

Tesis de Ingeniería Industrial. San José, C.R:

F. ChongKan Ch. y M. Arce A, 1990.

CHA VARRIA ROCHA, MANUEL; ROJAS FANDINO, SYLVIA y SEAS OSES, VILET.

Fluctuación de personal dentro de la industria de maquila textil exportadora

Tesis de Ingeniería Industrial. San José, C.R:

M. Chavarría R. et al., 1990.

BONILLA RODRIGUEZ, Rj FLORES SOTO, MANUEL Y MONGE VARGAS, HUGO.

Optimización del uso de los recursos en el departamento de construcción de equipo y repuestos GTE-Sylvania.

Tesis de Ingeniería Industrial. San José, C.R:

R Bonilla R et al., 1990.

CARMONA QUESADA, RONY y MANSO SAYAO, PAULO.

Análisis del proceso administrativoyplaneamiento estratégico del Departamento de clubes y crédito de Lacsa.

Tesis de Ingeniería Industrial. San José, C.R:

R Carmona Q. y P. Manso S., 1990.
ZAMORA AMADOR, ADRIANA

Propuesta de un modelo para la estructura orgánica de la U.CR

Tesis de Ingeniería Industrial. San José, C.R:

A Zamora A, 1990.

\section{ACUJ'JA PATIJ'JO, MANUEL Y VARGAS VILLALO-} BOS, RAMON ELIAS.

Diagnóstico y diseño conceptual de una base de datos para el manejo de la información del banco de recursos humanos dedicados a las acciones formativas en el Instituto Nacional de Aprendizaje

Tesis de Ingeniería Industrial. San José, C.R:

M. Acuña P. y RE. Vargas V., 1990.

GUTIERREZ ROJAS, PAUL; MONGE SALAS ROBERTyULATERODRIGUEZ, CARLOS.

Optimización del funcionamiento de la planta de servicios de la División Automotriz de COOPESA RL.

Tesis de Ingeniería Industrial. San José, C.R:

P. Gutiérrez R et al., 1990.

SCOTT WRIGHT, WILLIAM Y ZAMORA PEÑALBA,SANDRA.

Manual de procedimientos para riesgo químico para la Cruz Roja Costarricense

Tesis de Ingeniería Industrial. San José, C.R:

W. Scott W. y S. Zamora P., 1990.

\section{MORA KOPPER, GUILLERMO.}

Estudio de factibilidad para la implantación de una planta procesadora de broza de café

Tesis de Ingeniería Industrial. San José, C.R:

G. Mora K, 1990.

CASTRO G., LEDA Y ROTSCHILD, RANDALL.

Diagnóstico y bases del diseño de un sistema de información para el Centro de Evaluación Académica de la U.CR

Tesis de Ingeniería Industrial. San José, C.R:

L. Castro G. y R Rotschild, 1990.

SEGNINI TORRES, ALVARO; CHACO N ARAYA, JORGE HERNAN Y SOTO VIQUEZ, LUIS ROBERTO.

Evaluación e incremento de la productividad en LACSA Tesis de Ingeniería Industrial. San José, C.R:

A Segnini T. et al., 1990.

GARITA RODRIGUEZ, EUGENIAj JENKINS CORONAS, MANRIQUE y ROBERT BARRANTES, FEDERICO.

Sistema presupuestario del Hospital Calderón Guardia Tesis de Ingeniería Industrial. San José, C.R:

E. Garita R, 1990.

FERNANDEZ CABRERA, JOSE LUIS y SOLERA OSBORNE, JUAN MARIA .

Marco financiero para el desarrollo de empresas de alta tecnología en Costa Rica

Tesis de Ingeniería Industrial. San José, C.R: 
J. L. Fernández C. y J. M. Solera O., 1990.

FONSECA ZUÑIGA, SUSAN y JIMENEZ JIMENEZ, JEANNEITE.

Desconcentración de la C.C.S.S. en las áreas de recursos humanos, financieros y materiales

Tesis de Ingeniería Industrial. San José, C.R:

S. Fonseca Z. y J. Jiménez J., 1990.

\section{MONGE UNFRIED, YERICHy MORELLI C., EUGE-} NIO.

Estudio integral de adquisición y almacenamiento de materiales en CORTEL

Tesis de Ingeniería Industrial. San José, C.R:

Y. Monge U.y E. Morelli C., 1990.

PERALTA, JAIME F.

Certificación de calidad para el sector de cuero y calzado Tesis de Ingeniería Industrial. San José, C.R:

J. F. Peralta, 1990.

BARLEY GA YLE, DUNIA y MURILLO VALERIO, OVIDIO.

Diagnóstico de la capacidad de prestación de servicios de laU.C.R

Tesis de Ingeniería Industrial. San José, C.R:

D. Barley G. y O. Murillo V., 1990.

\section{ACUÑA NAVARRO, SONIA YRIVERA FOURNIER,} JAVIER

Estudio y análisis del sistema de suministros de Peribási$\cos$ S.A

Tesis de Ingeniería Industrial. San José, C.R:

S. Acuña N. y J. Rivera F., 1990.

\section{CANO ULATE, CLARKy LAURENT KAUFFMANN} JOHN

Diseño de un sistema de desarrollo de proveedores en la Gerencia de Desarrollo Industrial (RECOPE)

Tesis de Ingeniería Industrial. San José, C.R:

C. Cano U. y J. Laurent K, 1990.

\section{SANCHEZ ESQUIERDO, MARLON y SOLANO TO-} RRES, ALEX ENRIQUE.

Procedimiento para la detección de necesidades de entrenamiento dentro del marco del programa de Reconversión Industrial

Tesis de Ingeniería Industrial. San José, C.R:

M. Sánchez E. y A E. Solano T., 1990.

CASTRO BADILLA, MARCO Y ALVARADO VILLEGAS, PAULO.

Estudio de factibilidad para la industria Santa Juana

Tesis de Ingeniería Industrial. San José, C.R:

M. Castro B. y P. Alvarado V., 1990.

BARRANTES A, MANRIQUE; GAMBOA H., ILEANA Y GUERRERO C., MAURICIO.

Estudio de factibilidad para instalar una planta de procesamiento y congelamiento de frutas para la exportación

Tesis de Ingeniería Industrial. San José, C.R:
M. Barrantes A et alt., 1990. GONZALEZ GARCIA, ISAIAS y UMAÑA GOMEZ, JAVIER

Análisis y rediseño de la clasificación de artículos y algoritmos de reposición considerando las modalidades de compra establecidas en RECOPE

Tesis de Ingeniería Industrial. San José, C.R:

I. González G. y J. Umaña G., 1990.

BALTODANO CHAMORRO, MARIAMALIA y COTO DOBLES, CATALINA

Disefio y adaptación de la metodología de trabajo denominada "Justo a Tiempo" en Sintéticos S.A. Tesis de Ingeniería Industrial. San José, C.R: M. Baltodano Ch. y C. Coto D., 1990.

HEMPEL JIMENEZ, SIGURD; LIZANO SOTO, PABLO Y ROCHA CASTRO, ALBERRTO.

Propuesta de un sistema integrado de información para la producción .enla Oficina de Publicaciones de la U.C.R Tesis de Ingeniería Industrial. San José, C.R:

S. Hempel J. et alt., 1990.

NANNE P., KATHLEEN; NAVARRO R, MARCELA yVINDAS C., SILVIA.

Estudio de factibilidad técnica económica de la instalación de un beneficio de café en la zona de Chitaría, Turrialbalba

Tesis de Ingeniería Industrial. San José, C.R:

K Nanne P. eralt., 1990.

MAS GARCIA, ANA VELLA Y LEIV A MADRIGAL, LUIS FELIPE.

Factibilidad técnica y económica de la implantación de un modelo comunitario de eliminación de desperdicios orgánicos en la EARTH

Tesis de Ingeniería Industrial. San José, C.R:

A Mas G. y L. P. Leiva M., 1990.

CALDERONFEDULLO, JOSE RODRIGO.

Evaluación del modelo de atención médica ambulatoria por capacitación en el Centro Integrado de Barba Tesis de Ingeniería Industrial. San José, C.R:

J. R Calderón P., 1990.

MORALES GARCIA, ALVARO.

Desarrollo de una metodología de análisis, redisell.o e implantación de sistemas de control de inventarios. Tesis de Ingeniería Industrial. San José, C.R:

A Morales G., 1990.

FERNANDEZ MONTALTO, ARNOLDO y CALDWELL MARIN, ELDON.

Análisis y desarrollo de un sistema de información para el control de la producción de la gerencia de manufactura e ingeniería de la empresa $3 \mathrm{M}$ Costa Rica S.A Tesis de Ingeniería Industrial. San José, C.R:

A Pernández M. y E. Caldwell M., 1990. 
MAJCHEL P., AMIN y SABORIO P., ENRIQUE. Estudio de factibilidad técnico-económico de una planta productora de cajas de cartón y su disefio integral de planta.

Tesis de Ingeniería Industrial. San José, C.R:

A. Majchel P. y E. Saborío P., 1990.

\section{ANDRESJACOME,ALVARODEJESUSYSEQUEI-}

\section{RA SOLIS, LUIS ALBERTO.}

Estudio de áreas y procedimientos que afectan la productividad en la empresa Hortifruti S.A.

Tesis de Ingeniería Industrial. San José, C.R:

A. J. Andrés J. y L. A. Sequeira S., 1990.

\section{BOGANTESHERRERA, BEATRIZ Y RAMIREZVE-}

GA, ANA PATRICIA.

Estudio integral del laboratorio de soluciones parentales (C.C.S.S.).

Tesis de Ingeniería Industrial. San José, C.R:

B. Bogantes H. y A. P. Ram\{rez V., 1990.

\section{HERNANDEZ HERRERA, SERGIO $Y$ HERRERA} MUÑOZ, RANDALL.

Estudio integral del laboratorio de productos farmaceúticos de la c.C.S.S. para el mejoramiento de la productividad

Tesis de Ingeniería Industrial. San José, C.R:

S. Hernández H. y R Herrera M., 1990.

\section{BALTODANO CH., LUIS DIEGO YZAMORA RIOS, ARNOLDO.}

Diseño de un modelo administrativo para un centro de capacitación de personal de empresas relacionadas con el servicio de transporte aéreo.

Tesis de Ingeniería Industrial. San José, C.R:

L.D. Baltodano Ch. y A. Zamora R, 1990.

MENDEZ VEGA, JUAN CARLOS; PEÑA BOHORQUEZ, GLORIA S.; TORRES RODRIGUEZ, IV ANNIA YWARD WITTINGHAN, JAVIER

Análisis de la problemática existente en la consulta externa de las clínicas mayores de la C.C.S.S.

Tesis de Ingeniería Industrial. San José, C.R:

J. C. Méndez V. et alt., 1990.

ALVAREZ VELAZQUEZ, JOSE; GARRO HERRERA, DINORAH Y ZA VALETA OCHOA, OSCAR

Desarrollo de un modelo de sistema quirúrgico en el Hospital San Juan de Dios

Tesis de Ingeniería Industrial. San José, C.R:

J. Alvarez V. et alt., 1990.

\section{ZUÑIGA LEITON, ILEANA.}

Diseño de un sistema de información para la evaluación de la obra civil en la c.C.S.S.

Tesis de Ingeniería Industrial. San José, C.R:

I. Zúniga L., 1990.
ROJAS H., IV AN YSEGURA, JOHNNY.

Estudio integral de la empresa Embases Desechables S.A. tendiente al diseño y mejoramiento de la productividad

Tesis de Ingeniería Industrial. San José, C.R:

1. Rojas H. y J. Segura, 1990.

\section{DURAN CALVO, LUZESTHERyJARA VAZQUEZ,} JULIETA.

Disefio de un programa de aciión social para la Escuela de Ingeniería Industrial

Tesis de Ingeniería Industrial. San José, C.R:

L. E. Durán Calvo YJ. Jara V., 1990.

\section{ZA VALETA RAEZ, MARIA DEL PILAR}

Modelo de captación de recursos externos para la Facultad de Ingeniería de la Universidad de Costa Rica Tesis de Ingeniería Industrial. San José, C.R: M. P. Zavaleta R

LIZADO POVEDANO, VERNE.

Diseño de un sistema de control estadístico en procesos de fabricación de cables eléctricos.

Tesis de Ingeniería Industrial. San José, C.R:

V. Lizado P., 1990.

PONSECA BRID, RODRIGO.

Sistema de información profesional para jovenes yadultos que se insertan por primera vez en la formación profesional

Tesis de Ingeniería Industrial. San José, C.R:

R Fonseca B., 1990.

ACUÑA CORDERO, OSCAR

Organización delsistema administrativo de la Escuela de Ingeniería Industrial

Tesis de Ingeniería Industrial. San José, C.R:

O. Acuña C., 1990.

\section{NAVARRO GARITA, MARIO ALBERTO.}

Diseño de un sistema de planea miento y operación para el departamento de arquitectura y mantenimiento del INA

Tesis de Ingeniería Industrial. San José, C.R:

M. A. Navarro G., 1990.

GAMBOA CASTRO, ANA LILIANA Y ZUÑIGA PEREIRA, ILEANA MARIA.

Estudio del sistema de enseñanza aprendizaje de la Escuela de Ingeniería Industrial

Tesis de Ingeniería Industrial. San José, C.R:

A. L. Gamboa C. y I. M. Zúñiga P., 1990.

\section{RESUMENES.}

ALV AREZ VELAZQUEZ, JOSE; GARRO HERRERA, DINORAH y ZA VALETA OCHOA, OSCAR

Desarrollo de un modelo de sistema quirúrgico en el Hospital San Juan de Dios

Tesis de Ingeniería Industrial. San José, C.R:

J. Alvarez V. et alt., 1990. 
Los hospitales están definidos como las instituciones que brindan un apoyo importante a la comunidad en lo que a salud se refiere, debido a esto, los aportes que se pueden dar, enfocados en el mejoramiento de los servicios de salud van a favorecer a ésta.

El Hospital San Juan de Dios (H.SJ.D.) está enmarcado en este contexto, de ahí la inquietud de haber designado una serie de estudios que van a mejorar su funcionamiento.

La presente investigación se desarrolla con base en "El Enfoque de Sistemas"; Filosofía empleada ampliamente en la actualidad; para dirigir la estructuración global de las actividades del procesamiento de datos, necesarias para satisfacer las necesidades de información en las organizaciones modernas.

El anáslisis de sistemas se utiliza ¡:Qmo técnica general para resolver problemas, lo mismo como método para desarrollar sistemas de información.

Aplicando los conocimientos del Enfoque de Sistemas, se realizaron estudios estadísticos, entrevistas y revisiones bibliográficas para determinar y describir detalladamente las relaciones de cada uno de los subsistemas del sistema H.SJ.D., respecto al Sistema de Unidad Quirúrgica en estudio.

En el capítulo 1, se presenta la Hipótesis planeada, el objetivo general y los objetivos específicos con la metodología utilizada para el cumplimiento de los mismos.

El Capítulo II, contiene una introducción a la Filosofía de Sistemas, analizando cada Subsistema y su relación con el Sistema en estudio.

El Capítulo III, presenta el análisis del Sistema de Unidad quirúrgica, detallando cual es su porcentaje de utilización.

En la segunda parte de la investigación se diseña el modelo de unidad quirúrgica tomando en cuenta todas las variables estudiadas; y por último se desarrollan las conclusiones de la investigación.

Integrantes del panel: Ing. Carlos Quesada, Ing. Manuel Montoya, Ing. Roberto Barrantes, Dr. Luis B. Sáenz y Dr. Edgar Cabezas.

PERALTA, JAIME F

Certificación de calidad para el sector de cuero y calzado Tesis de Ingeniería Industrial. San José, C.R:

J. F. Peralta, 1990.

En este momento, Costa Rica está atravesando una etapa de transición, que afecta directamente al sector productivo del país, y por el cual hace necesario tomar decisiones rápidas y concretas en cuanto al mejoramiento de productividad $\mathrm{y}$, por ende, en una de las principales herramientas de ésta, la calidad.

Dentro del mejoramiento continuo de la calidad, la Certificación de Calidad viene a ser un instrumento de mejoramiento de ésta $\mathrm{y}$, el presente proyecto propone crear y conformar un mecanismo de Certificación de Calidad, basándose en las necesidades reales del país, específicamente de los industriales dentro del área de cuero y calzado.

Parte de la etapa de transición la componen el ingreso de Costa Rica al GATTysus consecuencias, un programa de ajuste estructural y un programa de reconversión industrial. Estos factores que influyen directamente en dicha etapa, tienden a mejorar la competitividad de los productos costarricenses, tanto en el mercado nacional como en el mercado internacional.

El aprovechamiento de los recursos que los mencionados programas tienen, depende propiamente de la agilidad, versatilidad y flexibilidad que debe de adoptar el producto del país, para convertirlos en recursos positivos $y$, por 10 tanto, en beneficios directos.

Integrantes del panel: Ing. Arman'do Castro, Ing. Guillermo Vargas, Ing. Ileana Aguilar, Lic. Jorge Chacón, Lic. Rolando Leiva e Ing. Roy Zúñiga.

\section{ESCUELA DE CIENCIAS DE LA COMPUTA- CION E INFORMATICA}

\section{Y11Semestres de 1990}

\section{RESUMENES}

\section{BOZAARAYA, ENRIQUE; PIEDRAARRIETA,RO-} NALD Y UGALDE RESENTERRA, SERGIO. Implementación del Estandar de Graficación: Graphical Kernel System (G.K.S.).

Tesis de Ciencias de la Computación e Informática. San José, C.R:

E. Boza A, et al., 1990.

En las dos últimas décadas la importancia y difusión de la disciplina de las Ciencias de la Computación denominada Graficación por Computadora, han sido inusitadas. Cada día son más numerosas y sofisticadas las aplicaciones que procuran facilitar la interacción computadora ser humano, mediante el método más dinámico y efectivo que se conoce a la fecha: la graficación.

Nuestro país no escapa a esta influencia y es así como desde ya hace bastante tiempo, se ha mostrado a nivel académico y comercial, gran interés por la Graficación por Computadora. Sin embargo, el tema de la Graficación no ha sido atacado en forma estructurada, la mayoría de los esfuerzos en este campo se han concentrado en aplicaciones de uso específico, sin un lenguaje común que las relacione y por ende sin la posibilidad de rescatar lo fundamental de un proyecto para la generación de otro. Definitivamente en nuestro país se ha hecho caso omiso de la tendencia general que en Graficación y en general en las Ciencias de la Computación, dirige el quehacer mundial: la estandarización. He ahí la razón de este Proyecto de Graduación, cuyo objetivo final es la implementación del paquete estándar para graficación "Graphical Kernel System" (GKS), sobre microcomputadores IBM PC o compatibles y utilizando ellenguajede programación Turbo Pascal 5.0.

El GKS fue escogido por la Organización Internacional para la Estandarización (ISO) en 1983, como el primer estándar internacional para la programación de aplicaciones gráficas.

Ofrece funciones para la generación y representación de gráficos, su estructuración o segmentación, la aplicación de transformaciones y rutinas para el procesamiento interactivo. Sin embargo, sus capacidades gráficas no son los únicos aspectos importantes del proceso que culmina 
con el GKS. Una ventaja aún más significativa es el hecho de que, por primera vez, se ha desarrollado una red metodológica para los muchos conceptos dentro de los gráficos por computadora. Esta se ha convertido enla base para un entendimiento $y$ una terminología común en la creación de sistemas de graficación, para el uso de los sistemas disponibles, para hablar acerca de graficación y para la enseñanza de los métodos, conceptos y aplicaciones de los gráficos por computadora.

La implementación resultante del proyecto permite concretaresta red metodológica, mediante el diseño deta-lIadoyla programación de las funciones definidas en el GKS. Para el establecimiento de este ambiente de desarrollo, se consideraron factores como el"hardware" sobre el que se implementó el sistema, el "software" base para el desarrollo, el lenguaje de programación y elpotencial necesario (nivel de la implementación) para abarcar la gran mayoría de las aplicaciones gráficas a desarrollar. Además, se incluye una extensa documentación interna y externa y algunos programas de demostración que utilizanel GKS.

Director de Proyecto: Lic. Allan Calderón Castro.

\section{NUÑEZ ALFARO, LUIS y RODRIGUEZ MONGE,} ARNOLDO.

Opciones para la Implementación de una Red Computadorizada como Parte del Sistema Nacional de Información en Ciencia y Tecnología.

Tesis de Ciencias de la Computación e Informática. San José, C.R:

L. Núñez A y A Monge R, 1990.

El gobierno de Costa Rica ha impulsado durante más de ocho años la creación de centros de información especializada (CIEs), a través de los cuales se brinda información $y$ asistencia técnica en las áreas científico tecnológicas. Este impulso ha culminado con la obtención del préstamo CONARE-CONICIT/BID, para el fortalecimiento de la ciencia y tecnologia en el país por medio de la ejecución de varios programas, siendo uno de ellos la creación de una Red de Información en Ciencia y Tecnología basada en la tecnología informática y de telecomunicaciones.

El proceso de elaboración y presentación de las opciones tecnológicamente disponibles en la actualidad para la conformación de la red de computadores, parte medular del proyecto, fue enriquecido con los resultados de un estudio teórico y de trabajo de campo; enmarcando los resultados dentro de los aspectos técnicos, operativos y financieros.

Del estudio yanálisis de los requerimientos de comunicación encontrados y de las restricciones existentes en el país, se concluye la necesidad de considerar opciones para una "red en maya", de donde surge la Red Privada como la opción que mejor satisface en cuanto a los aspectos técnicos.

Esta red se puede realizar utilizando, por ejemplo, el protocolo X.25 de la CCITT, para lo cual solo se requiere una línea entre cada nodo, más 12 modems. Esta decisión es refonada por los resultados del estudio de los aspectos operativos y financieros.

El desarrollo satisfactorio de la red de computadores depende no solo de aspectos tecnológicos sino también organizativos, presupuestarios y humanos, entre otros, y para cumplirlos se requiere un espíritu de colaboración por parte de todos los miembros participantes en la red. Por la parte informática y computacional, el reto de este proyecto ha sido plantear un diseño conceptual en una situación donde no se encuentran definidos completamente los componentes de la red.

\section{ESCUELA DE INGENIERIA MECANICA}

\section{Semestre de 1990}

\section{HARTMAN LANG, I-IARRY.}

Sistema de enfriamiento para evitar Alopecia inducida por la quimioterapia.

Tesis de Ingeniería Mecánica. San José, C. R:

H. Hartman L., 1990.

\section{RAMIREZ CORTES, JUAN CARLOS.}

Determinación de los parámetros ambientales de diseño para sistemas de aire acondicionado en Liberia

Tesis de Ingeniería Mecánica. San José, C. R:

J. C. Ramírez C., 1990.

\section{QUINTANA ORTIZ, MAURICIO.}

Modificación de la boquilla e implementación de un dispositivo desviador de flujo en turbinas Banki. Tesis de Ingeniería Mecánica. San José, C. R: M. Quintana O., 1990.

\section{ESPELETA VILLALOBOS, ROLANDO.}

Efectos del uso de intercambiadores de calor en un sistema de refrigeración.

Tesis de Ingeniería Mecánica. San José, C. R:

R Espeleta V., 1990.

\section{SPOERL HERNANDEZ, LOTHAR}

Estudio de opciones y recomendaciones para el control y protección de minicentrales hidroeléctricas.

Tesis de Ingeniería Mecánica. San José, C. R:

L. Spoerl H., 1990.

\section{GUTIERREZ FLORES, ERICK FRAN.}

Estudio de prefactibilidad para instalar un sistema de proteccion contra incendio en la terminal aérea del Aeropuerto Internacional Juan Santamaría.

Tesis de Ingeniería Mccánica. Sall José, C. R:

E.F. Gutiérrez F., 1990.

\section{CHI A YI, ROXANA}

Diseño de la transmisión hidrostática para una barredora. Tesis de Ingeniería Mecánica. San José, C. R: R Chi A, 1990

\section{ALVARADO MONTOYA, HUGO.}

Sustitución de gasolina y diesel por LPG en el Sector Transporte Costarricense.

Tesis de Ingeniería Mecánica. San José, C. R: H. ALV ARADO M., 1990. 
BOGARIN GONZALEZ, OSCAR ROLANDO.

Programa computadorizado para el cálculo de la carga térmica en sistemas de aire acondicionado.

Tesis de Ingeniería Mecánica. San José, C. R:

O.R Bogarín G., 1990.

\section{Semestre de 1990}

\section{TABASH BLANCO ADEMIR}

Programa de Mantenimiento Preventivo para el Equipo No.18 de RECOPE.

Tesis de Ingeniería Mecánica. San José, C. R:

A. Tabash B., 1990.

\section{INDUNI LOPEZ, HUGO.}

Auditoría energética del sistema de vapor del Plantel El Alto (RECOPE).

Tesis de Ingeniería Mecánica. San José, C. R:

H. Induni L., 1990.

ALVARADO VILLALOBOS, JUAN de la CRUZ. y AJUN LOPEZ, LUIS RODOLFO.

Programa de cálculo para el diseño y simulación de separadores de vapor empleados en pozos geotérmicos.

Tesis de Ingeniería Mecánica. San José, C. R:

J. de la C. Alvarado V. y L.R Ajún L., 1990.

\section{SILES CHACON MANUEL ALBERTO y SOLIS RO-} JAS, CARLOS .MANUEL.

Diseño y construcción de una turbina Pelton.

Tesis de Ingeniería Mecánica. San José, C. R:

M. A. Siles Ch. y C. M. Solís R, 1990.

\section{JACKSON QUIROS HERBERT.}

Situación actual del Ingeniero Mecánico, graduado de la Universidad de Costa Rica, en el Sector Privado. Tesis de Ingeniería Mecánica. San José, C. R: H. Jackson Q., 1990.

BEJARANO GUTIERREZ, OSCAR H.

Diseño y construcción de una máquina RR MOORE de Fatiga.

Tesis de Ingeniería Mecánica. San José, C. R:

O. H. Bejarano G., 1990.

GUEV ARA RODRIGUEZ, OTTO y VILLALOBOS CALDERON, RIGOBERTO.

Estudio sobre la construcción de engranajes en Costa Rica.

Tesis de Ingeniería Mecánica. San José, C. R:

O. Guevara R y R Villalobos C., 1990.

\section{ROLDAN CHACO N, FERNANDO.}

Diseño de un sistema de aire acondicionado para las áreas de medicina nuclear y el laboratorio de hormonas y endocrinología del Hospital Dr. Rafael A. Calderón Guardia. Tesis de Ingeniería Mecánica. San José, C. R: M. A. Roldán Ch., 1990.
LONGAN SANTONASTASIO, EDUARDO.

Tubo de desfogue para turbina Banki.

Tesis de Ingeniería Mecánica. San José, C. R:

E. Longan S., 1990.

\section{RESUMENES}

GUTIERREZ FLORES, ERICK FRAN.

Estudio de prefactibilidad para instalar un sistema de protección contra incendio en la terminal aérea del Aeropuerto Internacional Juan Santamaría.

Tesis de Ingeniería Mecánica. San José, C. R:

E. F. Gutiérrez F., 1990.

El objetivo fundamental de este trabajo es crear conciencia sobre la necesidad urgente de adoptar la "ingeniería de incendio" en Costa Rica. Esto es necesario dado que en los últimos años se ha incrementado la construcción de grandes obras y edificios, lo que hace imperativo contar con sistemas modernos, eficientes y seguros en los edificios que pueden presentar algún riesgo para la seguridad humana y material.

Se escogió el Edificio de la Terminal aérea del Aeropuerto Internacional Juan Santamría, para que sirva como modelo en el análisis y diseño de estos sistemas y para que el trabajo tenga una utilidad. El trabajo realizado permite obtener importantes conclusiones y recomendaciones para complementar y mejorar el aeropuerto.

La tesis comienza haciendo un breve estudio sobre la influencia del entorno socioeconómico y cultural en la decisión de invertir en equipos de lucha contra el fuego. Se constata que esos factores, el socioeconómico y el cultural, condicionan el desarrollo de la ingeniería de incendio en el país. También se constata que se obtienen beneficios en las instituciones o empresas que poseen sistemas contra incendio y cuyos gerentes y administradores son hábiles en la negociación de seguros.

En otro capítulo se describen brevemente varios sistemas utilizados en la protección contra incendio, entre ellos: seis tipos de sistemas de aspersión automáticos, gabinetes de mangueras, sistemas de espumas, sistemas de inundación total de halón 1301, halón 1211 y dióxido de carbono. Se hace énfasis en los sistemas de aspersión, dado que el sistema propuesto de protección contra incendio para el Aeropuerto es a base de agua.

También se evalúa el Aeropuerto en aspectos tales como: seguridad ysupervivencia, sistemas de aire acondicionado y ventilación, protección del equipo electrónico y computacional, rampa de abastecimiento de combustible, protección en equipos de extracción de humo y grasas de los equipos de la cocina comercial, protección de cintas grabadas y videocintas, los aspectos de diseño y construcción de la terminal aérea y el equipo existente de protección contra incendio. De esa evaluación se concluye que es recomendable hacer algunas remodelaciones y cambios en el edificio de la terminal antes de instalar un sistema de protección contra incendio. Eso es necesario pues, dado el crecimiento no planificado se han construido oficinas, divisiones, bodegas, etc. con materiales inadecuados, tóxicos y de alta combustivilidad. También se pudo observar que el edificio está desprotegido en un 75 
a $80 \%$ de su área total, no contando con extintores y mucho menos con gabinetes de mangueras.

Finalmente, se muestran algunos indicadoressocioeconómicos y costos del proyecto, de manera que sirvan como punto de partida para un análisis socioecomico y financiero del sistema de protección propuesto.

Director de Tesis: Ing. Jorge Ciudad Sánchez.

HARTMAN LANG, HARRY.

Sistema de enfriamiento para evitar Alopecia inducida por la quimioterapia.

Tesis de Ingeniería Mecánica. San José, C. R:

H. Hartman L., 1990.

Esta investigación se propuso encontrar los parámetros de diseño de un sistema de enfriamiento, para evitar la alopecia inducida por la quimioterapia como tratamiento del cáncer. La hipótesis consiste en establecer que la reducción de la temperatura del cuero cabelludo, en el momento de aplicar el tratamiento, reduce la pérdida del cabello. Lo anterior se debe a dos factores: a.- El enfriamiento produce una vasoconstricción en el folículo del pelo que reduce la cantidad de droga absorbida, b.- existe una dependencia de ciertas drogas para la asimilación celular.

El cuero cabelludo, con un espesor de $5 \mathrm{~mm}$., debe ser enfriado a un rango de 18-28 oC, hasta una profundidad de $2 \mathrm{~mm}$., para abarcar el folículo piloso.

Se aplica un método de diferencia finita unidimensional, con una linealización de la ecuación de difusión para la modelación de la cabeza, tanto en el estado estable como en el transitorio. Con el estado estable se obtienen las condiciones necesarias para conseguir la distribución de temperatura deseada, así como la pérdida de calor de la cabeza para dichas condiciones. Con el análisis del estado transitorio se obtiene el tiempo, previo a la inyección, en el que se debe aplicar el sistema.

Estos análisis producen los siguientes resultados: se debe hacer pasar una corriente de aire a $6 \mathrm{oC}$, con una velocidad de 11.27 mis alrededor de la cabeza para obtener las condiciones deseadas. Los tiempos de aplicación son de cinco minutos antes y treinta minutos después de la inyección.

El aire se hace recircular, con un $10 \%$ de aire fresco, por el gabinete y el casco, y es enfriado por un serpentín evaporador que consta de un banco de tubos alineados, con refrigerante 12 circulando en su interior.

Director de Tesis: Ing. Oscar H. Coto Chinchilla.

\section{SILES CHACON MANUEL ALBERTO YSOLIS RO-} JAS, CARLOS .MANUEL.

Diseño y construcción de una turbina Pelton.

Tesis de Ingeniería Mecánica. San José, C. R:

M. A. Siles Ch. y C. M. Salís R, 1990.

En este trabajo se hace un estudio teórico-práctico para llevar a cabo el diseño y la construcción de una turbina Pelton, que opera bajo una carga neta de $148 \mathrm{~m}$., un caudal de 35 US y una velocidad de giro de $1800 \mathrm{rpm}$.

Se recopilan, de obras confiables, los principales fundamentos del diseño, con los cuales se determinan las formas y dimensiones de cada componente de la turbina para su posterior construcción.

Elaborado el prototipo en su totalidad, se sometió a las pruebas respectivas para conocer las características de funcionamiento. Estas pruebas se realizaron a una altura constante de 20 metros. El caudal permaneció constante en algunas pruebas y en otras se varió mediante el inyector.

Las pruebas efectuadas permitieron determinar buenas características de operación y una eficiencia de un $71 \%$. Finalmente, se dan a conocer algunas recomendaciones de fabricación, materiales a utilizar y se plantea la posibilidad de construcción a mayor escala para aprovechar así los recursos hidráulicos existentes en el país.

Directores de Tesis: Ing. Glenn Dewey White e Ing. Manuel A. Murillo Sánchez.

\section{BEJARANO GUTIERREZ, OSCAR H.}

Diseño y construcción de una máquina RR MOORE de Fatiga.

Tesis de Ingeniería Mecánica. San Jos6, C. R:

O. H. Bejaraoo G., 1990.

Este trabajo tuvo como objetivo el diseño y construcción de una máquina de fatiga de flexión invertida, de acuerdo con una modifiCación de la RR Moore, para ser utilizada como equipo' de laboratorio en la Escuela de Ingeniería Mecánica.

La máquina construida está diseñada para fallar probetas de una amplia gama de aceros, permitiendo la aplicación de momentos flectores puros de entre 9.1 y $83.6 \mathrm{~N}-\mathrm{m}$.

La fuente motriz es un motor universal de $10000 \mathrm{rpm}$ con un control electrónico de velocidad. El conteo de los ciclos se hace por medio de un sensor fotoel6ctrico.

La carga se aplica por el pincipio de brazo de palanca con pesas que se pueden deslizar para calibrar el momento requerido. Los ejes principales se alojan en cajas de cojinetes lubricados con grasa, mejorando la seguridad y mantenimiento de la máquina. La máquina procura semiautomatizar las operaciones de inicio y fin de cada prueba. La máquina experimentó, durante la etapa de prueba, un problema de vibración, el cual se atribuyó a deficiencias en la precisión del maquinado de los ejes y sus mordazas. Para efectos prácticos se considera que el grado de ruido yvibración es tolerable y que no afecta los fines para los cuales la máquina fue construida.

Se realizó un ensayo completo de fatiga (10 probetas), que dió como resultado un buen funcionamiento general de la máquina. Existe incertidumbre sobre los efectos de la vibración sobre los resultados.

Director de Tesis: lng. Alejandro Pacheco Malina.

\section{ESCUELA DE INGENIERIA QUIMICA}

\section{Y11Semestres de 1990}

Arce Rodríguez, Ileana.

ESTUDIO FACTIBILIDAD AMPLIACIONPLANTA

LECHE CABRA, P/PRODUCIR QUESO FRESCO Y.

Tesis de Ingeniería Química. San Jos6, C.R:

1. Arce R, 1990. 
Borquet Barcía, Hafida.

TRATAMIENTO DESECHOS DE PLANTA DE CROMADO CON RECUPERACION DE COBRE $y$ NL

Tesis de Ingeniería Química. San José, C.R:

H. Borquet B., 1990.

Carmiol Herrera, Alexander.

ESTUD. FACTIB. PRODUCCION HARINA DE CONSUMO ANIMAL, CON DESECHOS DE CASCO Tesis de Ingeniería Química. San José, C.R:

A. Carmiol H., 1990.

Gutiérrez Herrera, José Raúl.

CARACTERISTICASDELPULPEO ORGANO SOLVENTE MODIFICADOR DE EUCALIPTUS SAL.

Tesis de Ingeniería Química. San José, C.R:

J. R Gutiérrez H., 1990.

Muño:GMoreira, Roy Alberto.

PROPIEDADES FUNDAMENTALES LEUCAENA

P. UTILIZ. EN PRODUCC. PA.

Tesis de Ingeniería Química. San José, C.R:

R A. Muñoz M., 1990.

Rueda González, Roberto.

TRATAMIENTO ANAEROBICO DE AGUAS DESECHO DEL CAPE, UTILIZ. REACFOR CON MA.

Tesis de Ingeniería Química. San José, C.R:

R Rueda G., 1990.

Salas Sarkis, Luis Federico.

CARACTERIZACION DE LA DIATOMITA GUANACASTECA COMO FILTRO AYUDA.

Tesis de Ingeniería Química. San José, C.R:

L. F. Salas S., 1990.

Vásquez Jiménez, Victor R

MANUALPIEVALUACION DESISTEMAS DESEGURIDAD E HIGIENE INDUSTRIAL EN PLA.

Director, Ing.Carlos Montero

Tesis de Ingeniería Química. San José, C.R:

V. R Vásquez J., 1990.

Vindas Evans, Jorge A.

SELECCION DE SISTEMAS DE TRATAMIENTO DE LAS AGUAS.

Tesis de Ingeniería Química. San José, C.R:

J. A. Vindas E., 1990.

Zelaya Goebel, Chester.

DISEÑO CONSTRUCCION y ANALISIS DE BIOREACTOR P/PRODUCCION ETANOLAPARTI.

Tesis de Ingeniería Química. San José, C.R:

Ch. Zelaya G., 1990.

Zolin Conce, Alfredo.

ESTUD. FACTIB. TECNICO-ECONOMICA P/INSTALACION PLANTA RECUPERACION CORMO.

Tesis de Ingeniería Química. San José, C.R:

A. Zolin C., 1990.
Hernández Orozco, Leticia.

OBT. NIVLS. ADEC. VARIAB. INTERV. DESTINTA-

DO PAPEL DE DESECHO FLOTACION.

Tesis de Ingeniería Química. San José, C.R:

L. Hernández O., 1990.

Lépiz Villegas, Tatiana.

EVAL. DE CAPAC. DEL EUCALPTUS SALIGNA $\mathrm{P}^{\prime}$ PROD. PAPEL LINER MEDIAN PULPE.

Tesis de Ingeniería Química. San José, C.R:

T. Lépiz V., 1990.

Meneses Obando, Grettel.

PROTOCOLO P' ESCOGER TRATAMIENTO QUIMICO OPTIMO SISTEMAS ENFRIAM. ABIERT.

Tesis de Ingeniería Química; San José, C.R:

G. Meneses O., 1990.

Montero Montero, José.

AUDITORIA ENERGETICA EN CAPE DORADO S.A.

Tesis de Ingeniería Química. San José, C.R:

J. Montero M., 1990.

Quesada Carboni, Joaquín A.

PURIFICACION BIOQUIMICA DEL AGUA UTILIZANDO LONES DE PLATA Y COBRE.

Tesis de Ingeniería Química. San José, C.R:

J. A. Quesada C., 1990.

Umaña Fernández, Pablo.

TRATAMIENTO ANAEROBICO DE VINAZAS.

Tesis de Ingeniería Química. San José, C.R:

P. Umaña F., 1990.

Valverde Gómez, Juan Carlos.

DETER DE LAS OPORTUN. DE CONSERVo DE ENERG. EN LAS EMPRESAS ZARAGOZA.

Tesis de Ingeniería Química. San José, C.R:

J. C. Valverde G., 1990.

Trigueros Fallas, Boris Manuel.

DESALADO OPTIMO DE PETROLEO CRUDO EN REFINERIA MOIN.

Tesis de Ingeniería Química. San José, C.R:

B. M. Trigueros F., 1990.

Jiménez Umaña, Jorge A.

ANALISIS TEC.-ECON. DE PROC. PIEL CONCENT. DEJUGOS ENLAPLANT. TICOFRU.

Tesis de Ingeniería Química. San José, C.R:

J. A. Jiménez U., 1990.

Vásquez Vásquez, Roberto.

ESTUDIO DE PREFACTIBILIDAD PARA LA PRO-

DUCCION DE CUERO RECONSTITUIDO.

Tesis de Ingeniería Química. San José, C.R:

R Vásquez V., 1990. 
Acón León, Victor.

EXPERIMENTACION EN UN ABSORBEDOR DE CONTACTO TURBULENTO.

Tesis de Ingeniería Química. San José, C.R:

V. Acón L., 1990.

Amén Chen, Carlos.

EST. PRE-FACTIB. RECUP. DE DImaDO CARBO-

NO DE UNA DESTILERIA ALCOHOLES.

Tesis de Ingeniería Química. San José, C.R:

C. Amén Ch., 1990.

Méndez Calderón, Armando.

DISEÑO SIST. DE TRATAM. DE AGUAS RESIDUALES PLANTA PRODUCC. DE PLAGUICID.

Tesis de Ingeniería Quími,ca. San José, C.R:

A. Méndez C., 1990.

\section{RESUMENES}

\section{ACON LEON, VICTOR}

Experimentación en un absorbedor de Contacto Turbulento.

Tesis de Ingeniería Química. San José, C.R:

V. Acón L., 1990.

El objetivo de este proyecto fué investigar el efecto de las diferentes variables que afectan al área interfacial de transferencia de masa en un absorbedor de contacto turbulento (ACT), utilizando el sistema sulfito de sodio-oxfgeno catalizado por sulfato de cobalto (II), la reacción es de primer orden. La columna empleada tiene $200 \mathrm{~mm}$ de diámetro, con una zona de operación de $1200 \mathrm{~mm}$, con una o dos etapas yconstruída en plástico acrílico transparente. Una solución de $60 \mathrm{~L}$ de sulfito de sodio $(0.45 \mathrm{M})$ se recicló continuamente a través de la columna. Se estudió el efecto de la siguientes variables: flujo de líquido (L), velocidad de aire (Ug), número de etapas (No ET.), porcentaje de área libre de rejilla retenedora $(\% \mathrm{~A})$, altura estática del lecho (Ha) y diámetro de partícula (dp). Se emplearon dos diseños experimentales, el primero un factorial fraccionado, 26-2y posteriormente un cuadrado latino 3 X3 duplicado.

A partir de los datos de los dos diseños experimentales y para el siguiente ámbito de condiciones de operación:

$3 \ll \mathrm{Ho} / \mathrm{dp})<10, \quad 160<\mathrm{Re}<970, \quad 420<\mathrm{Se}$ $<625,1.7<$ ast*dp $<20$

se obtuvo la siguie te correlación adimensional:

ast $* \mathrm{dp}=5.961 \mathrm{E}-\mathrm{T}(\mathrm{Ho} / \mathrm{dp}) \quad-1.064 *(\mathrm{Re}) \mathrm{O} .4 \mathrm{SO} * \mathrm{Se}) 2.427$ con un coeficiente de correlación de 0.988 y una desviación estándar de $56 \mathrm{~m} 2 / \mathrm{m} 3$, la cual es un $9 \%$ del promedio. Se recomienda para posteriores proyectos, el estudio en mayores ámbitos de la velocidad del aire, que la literatura señala como significativa, usando una sola etapa en la columna. El modo de fluidización corriente con el líquido burbujeo con unas pocas reformas, con lo que se puede realizar experimentación sobre aspectos de biotecnología que aprovecharía los conocimientos existentes en el país en el campo de la microencapsulación, método necesario en algunos procesos para tratar las partículas sólidas Director del Tesis: Ing. Manuel E. Molina.

UMAÑA FERNANDEZ, PABLO.

Tratamiento Anaeróbico de Vinazas.

Tesis de Ingeniería Química. San José, C.R:

Pablo Umafta F., 1990.

Se planteó como objetivo del proyecto estudiar el efecto del tiempo de retención hidráulico y de la razón de recirculación sobre la·eficiencia del tratamiento anaeróbico de vinazas en el ámbito termofílico en un reactor anaeróbico con mamparas (ABR). Para esto se utilizó un reactor a nivel de laboratorio, operando a una temperatura de $55^{\circ} \mathrm{C}$ con vinazas de la Fábrica Nacional de Licores (FANAL). Se varió el tiempo de retención hidráulico $(\mathrm{T})$ entre 6 días y 1 día, mientras que las razones de recirculación (R), definidas como flujo volumétrico recirculado entre flujo volumétrico de alimentación fresca, fueron de 2 y de 4 . Se obtuvo como resultado valores de la eficiencia de reducción de la nQO entre un 64\%, para un tiempo de retención de 6 días yR $=4$,yun $29 \%$ para $\mathrm{T}=1$ díayR $=2$.

Se concluyó que un aumento en la razón de recirculación tiene un efecto positivo sobre la eficiencia de tratamiento, siendo este efecto mayor conforme se disminuye el tiempo de retención hidráulico. Además, los datos experimentales se ajustaron a un modelo matemático que es:

$1 / \mathrm{Y}=0.0129+(0.0292-0.00365 \mathrm{R}) / \mathrm{T}(8)$

donde $Y$ es la eficiencia porcentual de reducción de la DQO, $\mathrm{R}$ es la razóñ de recirculación y $\mathrm{T}$ es el tiempo de retención hidráulico en días. El modelo predice una reducción máxima de la DQO de un 78. Se comprobó también que el reactor ABR permite formar lodo granular, al tratar vinazas en el ámbito termofílico.

Se recomienda modificar el disefto del reactor disminuyendo el volumen de la primera cámara con respecto al de las otras, para poder realizar una separación de fases microbiológicas por medio del control del tiempo de retención hidráulico, y separar la atmósfera de la primera cámara para evitar la inhibición de la degradación del propionato por alta presión parcial de hidrógeno, la cual es mayor en el ámbito termofílico que en el mesofílico. Por último, se recomienda escalar el experimento a nivel piloto para verificar el modelo matemático, estudiar su aplicabilidad a otros ámbitos de tiempo de retención hidráulico y de razón de recirculación, y darle seguimiento a la acumulación de inertes en el fondo del reactor y a la formación de natas en la superficie del líquido.

Director del Tesis: Ing. Fernando Silesky. 